OPEN ACCESS

Edited by:

Signe Altmäe,

University of Granada, Spain

Reviewed by:

Paweł Radwan,

Gameta Hospital, Poland

Nerea M. Molina,

University of Granada, Spain

${ }^{*}$ Correspondence:

Haixia Song

271905774@qq.com

orcid.org/0000-0002-5956-7349

${ }^{\dagger}$ These authors share first authorship

Specialty section: This article was submitted to

Reproduction,

a section of the journal

Frontiers in Endocrinology

Received: 12 September 2021

Accepted: 26 October 2021

Published: 23 November 2021

Citation:

Song $H$, Qin Q, Yuan C, Li H,

Zhang $F$ and Fan L (2021)

Metabolomic Profiling of Poor

Ovarian Response Identifies

Potential Predictive Biomarkers.

Front. Endocrinol. 12:774667.

doi: 10.3389/fendo.2021.774667

\section{Metabolomic Profiling of Poor Ovarian Response Identifies Potential Predictive Biomarkers}

\author{
Haixia Song ${ }^{1 \star}$, Qin Qin ${ }^{1 \dagger}$, Caixia Yuan ${ }^{1}$, Hong $\mathrm{Li}^{1}$, Fang Zhang ${ }^{2}$ and Lingling Fan ${ }^{1}$ \\ 1 Department of Reproductive Medicine, Shanxi Provincial People's Hospital, Taiyuan, China, 2 Department of Central \\ Laboratory, Shanxi Provincial People's Hospital, Taiyuan, China
}

Objective: To characterize the serum metabolomic profile and its role in the prediction of poor ovarian response (POR).

Patient(s): Twenty-five women with normal ovarian reserve (24-33 years, antral follicle count $[\mathrm{AFC}] \geq 5$, anti-Müllerian hormone $[\mathrm{AMH}] \geq 1.2 \mathrm{ng} / \mathrm{ml}$ ) as the control group and another twenty-five women with POR (19-35 years, AFC $<5, \mathrm{AMH}<1.2 \mathrm{ng} / \mathrm{ml}$ ) as the study group were collected in our study. The serum levels of the women in both groups were determined from their whole blood by untargeted liquid chromatography-mass spectrometry (LC-MS). Multivariate statistical analysis and cell signal pathways analysis were used to reveal the results.

Results: A total of 538 different metabolites were finally identified in the two groups. Tetracosanoic acid, 2-arachidonoylglycerol, lidocaine, cortexolone, prostaglandin H2,1naphthylamine, 5-hydroxymethyl-2-furancarboxaldehyde, 2,4-dinitrophenol, and Derythrulose1-phosphate in POR were significantly different from control as were most important metabolites in support vector machines $(p<0.05)$. Metabolomic profiling, together with support vector machines and pathway analysis found that the nicotinate and nicotinamide metabolism pathway, including L-aspartic acid, 6-hydroxynicotinate, maleic acid, and succinic acid semialdehyde, was identified to have significant differences in POR women compared to control women, which may be associated with ovarian reserve.

Conclusion: This study indicated that LC-MS-based untargeted metabolomics analysis of serum provided biological markers for women with POR. The nicotinate and nicotinamide metabolism pathway may offer new insight into the complementary prediction and therapeutic potential of POR. The functional associations of these metabolites need further investigation.

Keywords: poor ovarian response (POR), ovarian reserve, biomarkers, nicotinate and nicotinamide metabolism pathway, serum metabolomics 


\section{INTRODUCTION}

Poor ovarian response (POR), also called low ovarian response, is characterized by the pathological state of poor ovarian response to gonadotropin (GN) stimulation. At present, the diagnosis of POR is still unclear without unified criterion. Compared with Bologna standard (1), the Poseidon standard (2) wholly considers the age, ovarian reserve, and responsiveness of POR patients. This standard reduces population heterogeneity. In the process of ovulation induction of assisted reproductive technology (ART), some patients suffer from a low peak value of blood estrogen $\left(\mathrm{E}_{2}\right)$ on hCG administration day and a low number of retrieved oocytes, which are often accompanied by the large consumption of GN and a high cycle cancellation rate, which are collectively known as low ovarian response (POR).

How to improve the pregnancy rate and pregnancy outcome of POR patients is the main aim and difficulty of reproductive research. Current main diagnostic indexes of ovary reserve include age, basal serum follicular stimulating hormone (FSH), basic serum inhibin $B$, serum anti-Müllerian hormone (AMH), antral follicle count (AFC), basal ovarian volume, basal serum $\mathrm{E}_{2}$, and so on (3). The basal luteinizing hormone (LH)/FSH ratio and ovarian blood flow have also attracted attention $(4,5)$. A retrospective cohort study showed a novel mathematical model of true ovarian reserve assessment named the AAFA model based on predicted probability of POR: AMH, AFC, FSH, and age, in that order (6). However, how can we predict POR before these indicators change? We want to use metabolites in the serum as biomarkers in POR to infer whether these metabolites are related to ordinary diet or living environment, so as to provide more etiology guidance for the prevention or early treatment of POR.

Metabolomics is a global biochemical approach to test endogenous metabolites in biological systems and their dynamic changes in response to various factors (7-10). It has been widely used to predict and diagnose several diseases (11), especially in cancers to identify biomarkers in urine and serum among patients (12-14). Metabolomics has also been used to predict useful biomarkers in polycystic ovary syndrome (PCOS) (15-17).

There are few studies that identify biomarkers in serum in patients with POR by using metabolomics. We aim to characterize serum metabolomic profiling of POR patients using a liquid chromatography-mass spectrometry (LC-MS)based untargeted metabolomics approach, and subsequently to identify the potential biomarkers and metabolic pathway for the predictive significance and therapeutic potential of POR.

\section{MATERIALS AND METHODS}

\section{Sample Collection}

The clinical data of 50 women younger than 35 years old were collected from the Department of Reproductive Medicine of Shanxi Provincial People's Hospital between January 2021 and May 2021. The study was approved by the Ethics Committee of
Shanxi Provincial People's Hospital. All the participants provided written informed consent. Their hormones were examined and they underwent transvaginal ultrasonography, to determine the level of basal FSH, $\mathrm{LH}, \mathrm{E}_{2}$, progesterone (P), prolactin (PRL), testosterone (T), AMH, and AFC. According to different ovarian function status based on Poseidon Group 3, they were grouped into 25 women with normal ovarian reserve (as controls) and 25 women with POR.

The inclusion criteria for participants were as follows. Women aged 24-33 years, $\mathrm{AFC} \geq 5$, and $\mathrm{AMH} \geq 1.2 \mathrm{ng} / \mathrm{ml}$ were included as the control group. Women aged 19-35 years, AFC $<5, \mathrm{AMH}<1.2 \mathrm{ng} / \mathrm{ml}$ were included as the study group (Poseidon Group 3).

Women with the following conditions including endometriosis, a history of oophorectomy, congenital adrenal hyperplasia, thyroid disease, hyperprolactinemia, and other diseases affecting ovarian reserve were excluded.

Venous blood samples $(3 \mathrm{~mL})$ were taken on days 2-4 of the menstrual cycle after spontaneous or withdraw of progesterone in the presence of amenorrhea to determine the levels of hormones, after an overnight fast of at least $10 \mathrm{~h}$. AFC was calculated by counting the number of follicles in diameter with ranges from 2 to $9 \mathrm{~mm}$ in right and left ovaries by transvaginal ultrasonography. The plasma samples were isolated following centrifugation at $1,024 \mathrm{~g}$ for $10 \mathrm{~min}$. All of the clinical characteristics were examined at the laboratory of the Department of Reproductive Medicine of Shanxi Provincial People's Hospital. The samples were collected with a separating gel-promoting tube, and then centrifugated. The upper serums were stored at $-80^{\circ} \mathrm{C}$ until use.

\section{Sample Preparation}

All samples were centrifuged at $4^{\circ} \mathrm{C}$ for LC-MS analysis. A total of $100 \mu \mathrm{L}$ of each sample and $400 \mu \mathrm{L}$ of methanol $\left(-20^{\circ} \mathrm{C}\right)$ were transferred and mixed into $2 \mathrm{~mL}$ centrifuge tubes. The mixture was vortexed for $60 \mathrm{~s}$ and centrifuged at $4^{\circ} \mathrm{C}$ for $10 \mathrm{~min}$ at 12000 rpm. The supernatant was transferred from each sample into another $2 \mathrm{~mL}$ centrifuge tube and was concentrated until dry in a vacuum. In total, $150 \mu \mathrm{L}$ of 2 -chlorobenzalanine (4 ppm) $80 \%$ methanol solution was added to dissolve samples, and the supernatant was filtered through a $0.22 \mu \mathrm{m}$ membrane to obtain the prepared samples for LC-MS. A total of $20 \mu \mathrm{L}$ of each sample was taken for quality control (QC).

\section{LC-MS Analysis}

Chromatographic separation was accomplished in an Thermo Ultimate 3000 system equipped with an ACQUITY UPLC ${ }^{\circledR}$ HSS T3 $(150 \times 2.1 \mathrm{~mm}, 1.8 \mu \mathrm{m}$, Waters $)$ column maintained at $40^{\circ} \mathrm{C}$. The temperature of the autosampler was $8^{\circ} \mathrm{C}$. Gradient elution was carried out with $0.1 \%$ formic acid in water (C) and $0.1 \%$ formic acid in acetonitrile (D) or $5 \mathrm{mM}$ ammonium formate in water (A) and acetonitrile (B) at a flow rate of $0.25 \mathrm{~mL} / \mathrm{min}$. Injection of $2 \mu \mathrm{L}$ of each sample was done after equilibration. An increasing linear gradient of solvent B (v/v) was used as follows: 0 1 min, 2\% B/D; 1 9 min, 2\% 50\% B/D; 9 12 min, 50\% 98\% $\mathrm{B} / \mathrm{D} ; 12 \sim 13.5 \mathrm{~min}, 98 \% \mathrm{~B} / \mathrm{D} ; 13.5 \sim 14 \mathrm{~min}, 98 \% \sim 2 \% \mathrm{~B} / \mathrm{D} ; 14 \sim 20$ min, $2 \% \mathrm{D}$-positive model (14 17 min, 2\% B-negative model). 
The ESI-MSn experiments were executed on the Thermo Q Exactive Focus mass spectrometer with a spray voltage of $3.5 \mathrm{kV}$ and $-2.5 \mathrm{kV}$ in positive and negative modes, respectively. Sheath gas and auxiliary gas were set at 30 and 10 arbitrary units, respectively. The capillary temperature was $325^{\circ} \mathrm{C}$. The orbitrap analyzer scanned over a mass range of $\mathrm{m} / \mathrm{z} 81-1000$ for a full scan at a mass resolution of 70,000. Data-dependent acquisition (DDA) MS/MS experiments were performed with a HCD scan. The normalized collision energy was $30 \mathrm{eV}$. Dynamic exclusion was implemented to remove some unnecessary information in MS/MS spectra.

\section{Data Processing}

Raw data files were processed by the Proteowizard software (v3.0.8789). The XCMS package (v3.1.3) was used for peak identification, peak filtering, and peak alignment. We obtained a data matrix including mass to charge ratio $(\mathrm{m} / \mathrm{z})$, retention time (RT), and peak area (intensity). A total of 14881 peak indices in positive mode and 11947 peak indices in negative mode were observed between the samples. The data were exported to Excel for subsequent MetaboAnalystR analysis $(18-20)$.

\section{Metabolite Annotation and Pathway Analysis}

We conducted quality control (QC) and standardization of sample data. Histogram, thermogram, and unsupervised dimension reduction analysis (principal component analysis, PCA) were used to obtain the statistics of metabolite content. Supervised partial least squares discriminant analysis (PLS-DA) and supervised orthogonal partial least squares discriminant analysis (OPLS-DA) were used to measure the quality and reliability of the model by $\mathrm{R}^{2} \mathrm{Y}$ and $\mathrm{Q}^{2}$. Univariate analysis and machine learning [random forest, support vector machines (SVM)] were used for screening the importance of biomarkers by the area under the curve (AUC). Pathway analysis of characteristic metabolites (enrichment analysis, topology analysis, etc.) could also be used for identifying important metabolic pathways.

\section{Statistical Analysis}

The data of baseline clinical characteristics of all the subjects were analyzed by SPSS (version 23;SPSS Inc.). The KolmogorovSmirnov test (K-S test) was used to estimate normality of distribution. Normally distributed continuous variables were presented as means \pm standard deviations (SDs) and Student's t-test was used to compare the statistical difference between the two groups. Non-normally distributed numerical variables were presented by medians (25th to 75 th percentiles) and the nonparametric test was used to compare the statistical difference between the two groups. Two-sided $p$-values $<0.05$ were considered statistically significant.

\section{RESULTS}

\section{Baseline Characteristics of POR Patients and Controls}

A total of 50 subjects were enrolled in our study, including 25 control women with normal ovarian reserve (24-33 years, AFC $\geq 5, \mathrm{AMH} \geq 1.2 \mathrm{ng} / \mathrm{ml}$ ) and 25 study women with POR (19-35 years, $\mathrm{AFC}<5, \mathrm{AMH}<1.2 \mathrm{ng} / \mathrm{ml}$ ) diagnosed by Poseidon Group 3. The clinical characteristics are shown in Table 1. The mean age in the study was $29.36 \pm 3.42$ years, varying from 19 to 35 years. There were no statistically significant differences in age, $\mathrm{LH}, \mathrm{E}_{2}$, and $\mathrm{T}$ between the two groups $(p>0.05)$. Similar to another study, an increase in the levels of FSH but a decline in $\mathrm{AMH}$ and $\mathrm{AFC}$ in the POR group showed significant differences $(p<0.001)$. The POR group had a significantly lower PRL than the control group $(p<0.05)$, but all were in the normal range.

\section{Quality Assessment and QC of Serum Samples}

QC is one of the basic concepts of bioanalysis, which is used to ensure the repeatability and accuracy of data measured by omics. Using unsupervised PCA, we modeled the samples of each group, and then displayed the score plot (Figures 1A, B). This intra-group PCA analysis discarded the interference between groups, allowing us to more clearly observe the variation within the group and find possible outliers. Each point in the graph represents a sample, and the position of the point in the graph is determined by all metabolites in the sample. The ellipse in the figure is the $95 \%$ confidence interval calculated and drawn based on Hotelling's T2 metric. The sample fell outside the ellipse, suggesting that this point might be an outlier. However, we usually only considered deleting a sample when there were serious outliers, such as pulling the whole model out of shape.

TABLE 1 | Clinical characteristics of controls and POR group (median, $25^{\text {th }}-75^{\text {th }}$ ).

\begin{tabular}{|c|c|c|c|}
\hline Variables & Control group $(n=25)$ & POR group $(n=25)$ & $P$ value \\
\hline Age (y) & 29.00 (27.50-31.00) & 31.00 (26.50-34.00) & 0.287 \\
\hline FSH (miU/mL) & $7.14(6.49-7.53)$ & 20.96 (12.00-26.10) & $<0.001$ \\
\hline $\mathrm{LH}(\mathrm{miU} / \mathrm{mL})$ & 3.65 (3.08-4.62) & $4.30(3.18-12.34)$ & 0.200 \\
\hline Ez (pg/ml) & $35.00(27.50-39.41)$ & $40.00(28.07-71.15)$ & 0.053 \\
\hline $\mathrm{T}(\mathrm{ng} / \mathrm{dl})$ & 35.08 (25.15-44.41) & $29.00(19.5-37.75)$ & 0.233 \\
\hline $\mathrm{PRL}(\mathrm{ng} / \mathrm{m} 1)$ & $14.28(11.89-18.56)$ & $10.00(8.98-14.64)$ & 0.004 \\
\hline AMH (ng/ml) & $4.34(3.95-5.00)$ & 0.07 (0.04-0.53) & $<0.001$ \\
\hline $\operatorname{AFC}(n)$ & 18.00 (16.00-20.00) & $4.00(2.50-4.00)$ & $<0.001$ \\
\hline
\end{tabular}

FSH, follicle stimulating hormone; LH, luteinizing hormone; E2, estradiol; T, testosterone; PRL,prolactin; AMH, antimmüllerian hormone; AFC, antral follicle count. 


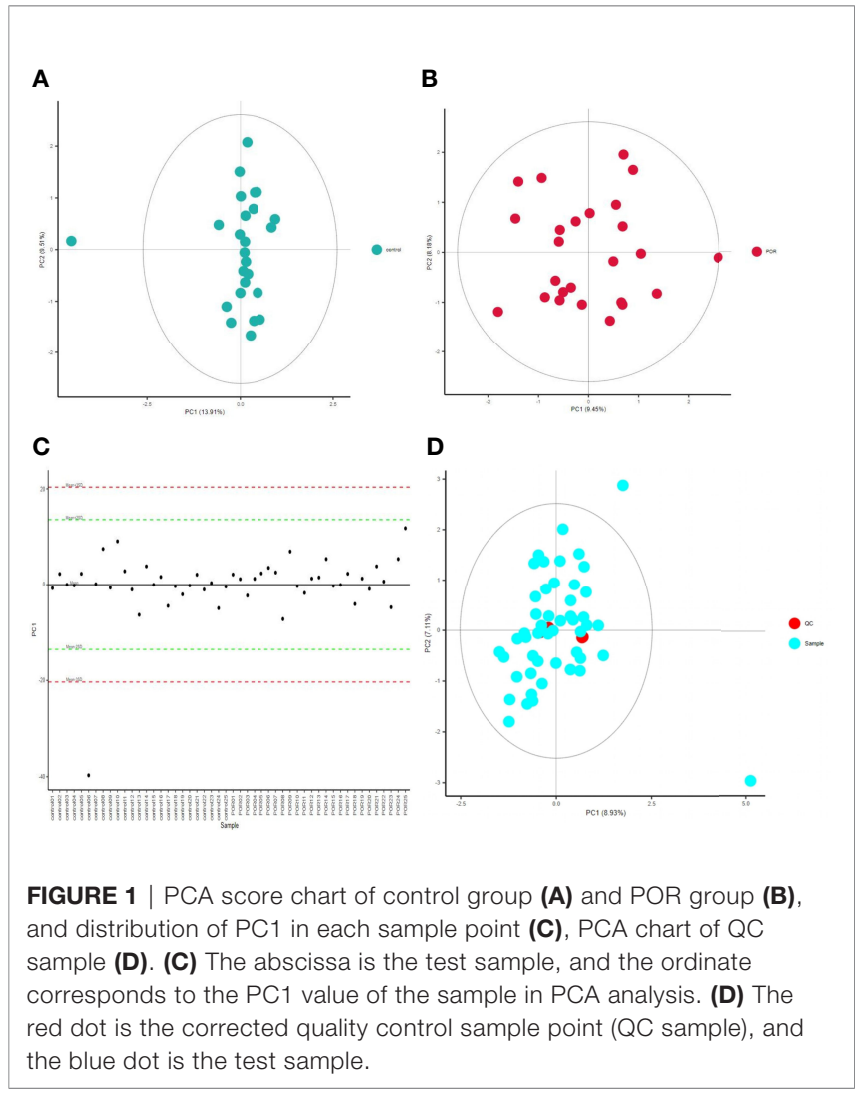

The distribution of PC1 values of all sample points was used to evaluate whether the sample preparation and measurement process was controllable, and most points would fluctuate up and down around the axis within 2 standard deviations above the mean (2SDs) (Figure 1C). In this process, the QC-RFSC algorithm was used to correct the characteristic signal peaks of each sample (each metabolite), and the correcting effect of each metabolite was recorded. After correcting the signal drift, the QC sample points were gathered together in the PCA diagram, which proved that the correcting effect was good (Figure 1D).

We used metabolite normalization for the purpose of making the mean and SD at the same level, and ensuring the later accuracy analysis of importance of all metabolites (Figure 2).

\section{Concentration Summary}

Overall, 538 different metabolites were finally identified in the two groups. The content percentage of each metabolite in each sample was calculated and visualized by a stacking column diagram, which can intuitively compare the differences of metabolite composition and structure between groups. Figure 3A shows the top 20 content percentage of metabolites from low to high, including (S)-beta-tyrosine, protoporphyrinogen IX, guanidinosuccinic acid, N-methyl-2-pyrrolidinone, (R)-4-hydroxymandelate, 4hydroxycinnamoylagmatine, 2-aminophenol, L-sorbose, phenylacetate, p-octopamine, L-methionine, gammaglutamylcysteine, L-glutamine, uric acid, piperidine, L-valine, oleic acid, L-tyrosine, 1-linoleoylglycerophosphocholine, and pyrrole-2-carboxylic acid.

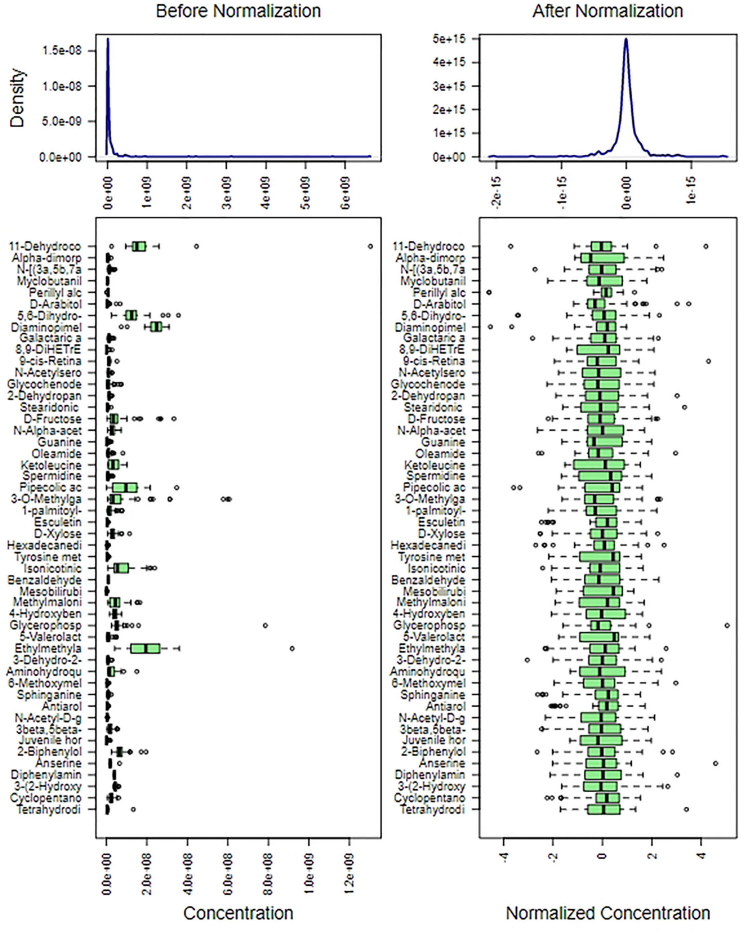

FIGURE 2 | Content distribution of each metabolite before and after normalization. The content distribution was represented by box plot, which was corresponded to outlier, minimum, lower quartile, median, upper quartile, maximum and outlier from left to right. The figure on the left shows the distribution before normalization, and the figure on the right shows the distribution after normalization.

The clustering analysis of samples by heatmap found the similarity between different products, and we constructed the clustering model of samples. The samples of different groups were clustered to different positions. Figure 3B indicated that the composition and structure of the top 30 metabolites were quite different between groups.

\section{Supervised Partial Least Squares Discriminant Analysis}

PLS-DA is supervised, that is, grouping information needs to be provided during analysis. PLS-DA looks for factors that can distinguish the grouping of samples to the greatest extent (factors can be understood as the weighted sum of all metabolites). Discriminant analysis encodes the discontinuous classification variable to be predicted as a latent variable, and the latent variable is continuous. In this way, a regression can be established between the explanatory variable and the latent variable, which can be solved by the theory of least square regression. Partial least squares discriminant analysis is an upgraded version of linear discriminant analysis, which is suitable for omics data with a large number of collinearity problems in explanatory variables. PLS-DA finds a linear regression model by projecting the prediction variables and observation variables into a new space (each dimension of the 
A

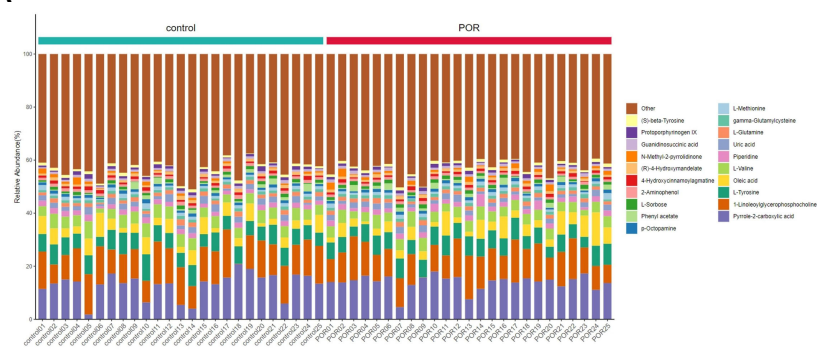

B

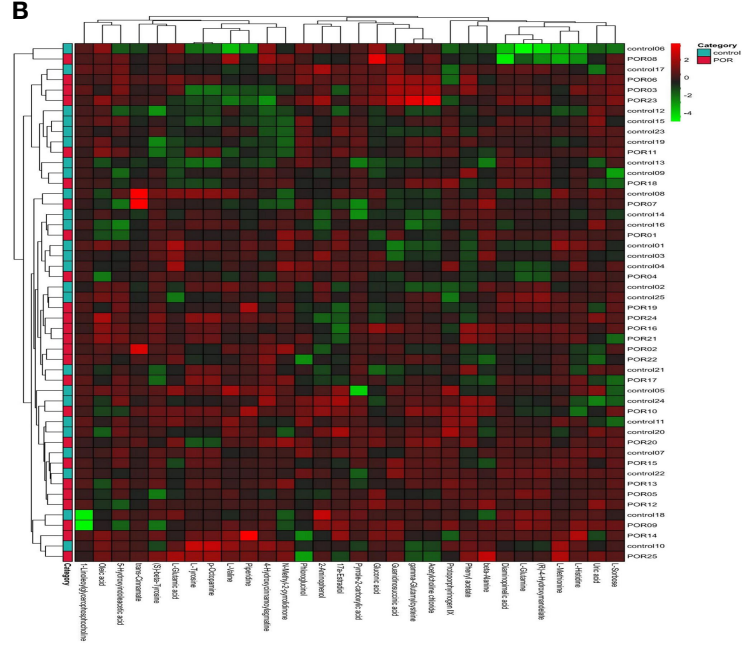

FIGURE 3 | Accumulation histogram (A) and heat-map (B) of differential metabolites visualized the variations of metabolite profiles of POR and controls. (A) The abscissa is the sample name, which is sorted according to the grouping order, and different grouping samples are marked with different colors. The ordinate represents the percentage content of each metabolite, and the column order of corresponding metabolites from top to bottom is consistent with the legend. (B) The vertical axis is the sample name information, and also includes the grouping information. The horizontal axis is the metabolite. The cluster tree at the top of the figure is the similarity cluster of the distribution of metabolites in each sample, and the cluster tree at the left is the sample cluster tree, and the heat map in the middle is the heat map of metabolite content. The relationship between color and Z-score is shown in the scale at the top right.

new space is independent of each other and there is no collinearity problem). As shown in Figure 4, we used the two factors with the best discrimination effect to draw the scatter diagram. The point clouds of the two groups were obviously distributed in different areas, which indicated the good discrimination effect of the PLS-DA model and the distant differences in the composition and structure of metabolites of the two groups.

\section{Supervised Orthogonal Partial Least Squares Discriminant Analysis}

Partial least squares discriminant analysis (PLS-DA) is also supervised, that is, grouping information is needed. OPLS-DA is an improvement of PLS-DA. The regression model is

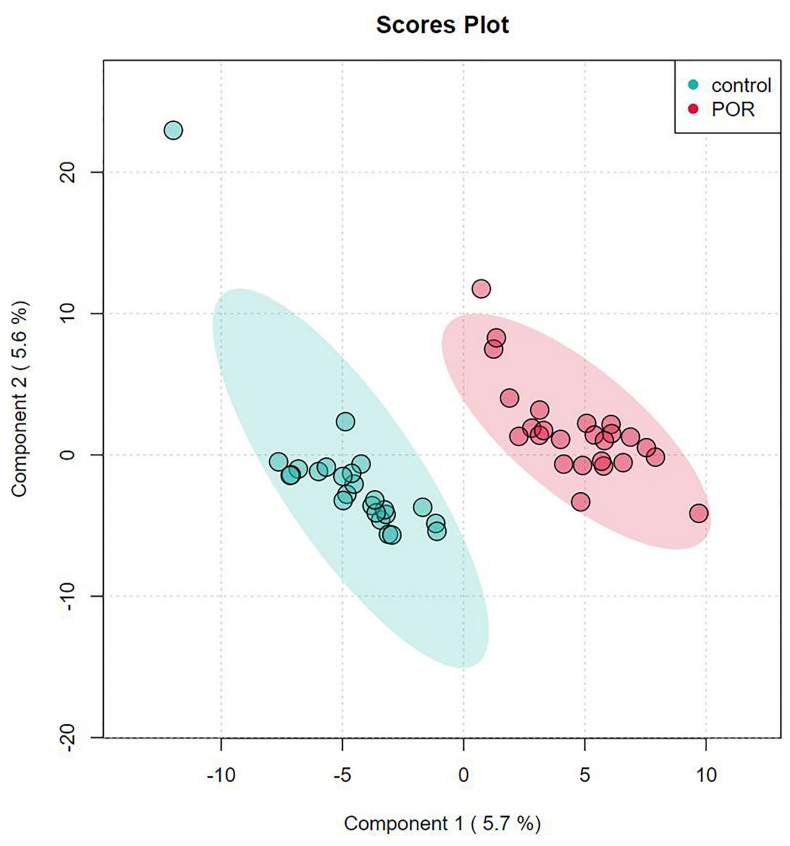

FIGURE 4 | Score plot analysis of PLS-DA of group of POR and controls. Each point corresponds to a sample, and the abscissa and ordinate are the values of the two factors with the best discrimination effect. Different groups are marked with different colors, and the area marked by ellipse is the $95 \%$ confidence area of sample points.

established between metabolome data and grouping information, and the model will filter out the information irrelevant to grouping. Compared with PLS-DA, the advantage of OPLS-DA is that only one component of metabolome data is used to predict grouping, while other components are used to describe orthogonal (unrelated) variation with the predicted component. In OPLSDA, we usually use $R^{2} Y$ and $Q^{2}$ to measure the quality and reliability of the model. The two values are usually close. The closer $\mathrm{R}^{2} \mathrm{Y}$ and $\mathrm{Q}^{2}$ are to 1 , the better the discrimination effect of the model. The OPLS-DA model achieved better separation $\left(R^{2} X=0.113, R^{2} Y=0.931, Q^{2}=0.612\right.$; Figure 5).

The process of discriminant analysis is used to find the metabolites that play the most important role (value importance projection, VIP) and can be used as biomarkers to distinguish different groups. As shown in Figure 6, the metabolites marked with the name in the yellow area were those with $p<0.05$ and VIP $>1$. These metabolites had significant differences between groups, which play an important role in OPLS-DA and should be paid attention to.

In the permutation test of OPLS-DA, we used $\mathrm{Q}^{2}$ as the test statistic and used the permutation method to obtain the random distribution of $\mathrm{Q}^{2}$ to confirm the stability and robustness of the model. As shown in Figure 7, the observed $Q^{2}$ value $\left(Q^{2}=0.612\right)$ was significantly greater than the random value, so the prediction ability of the model was significant and there should be metabolites with significant differences between groups. 


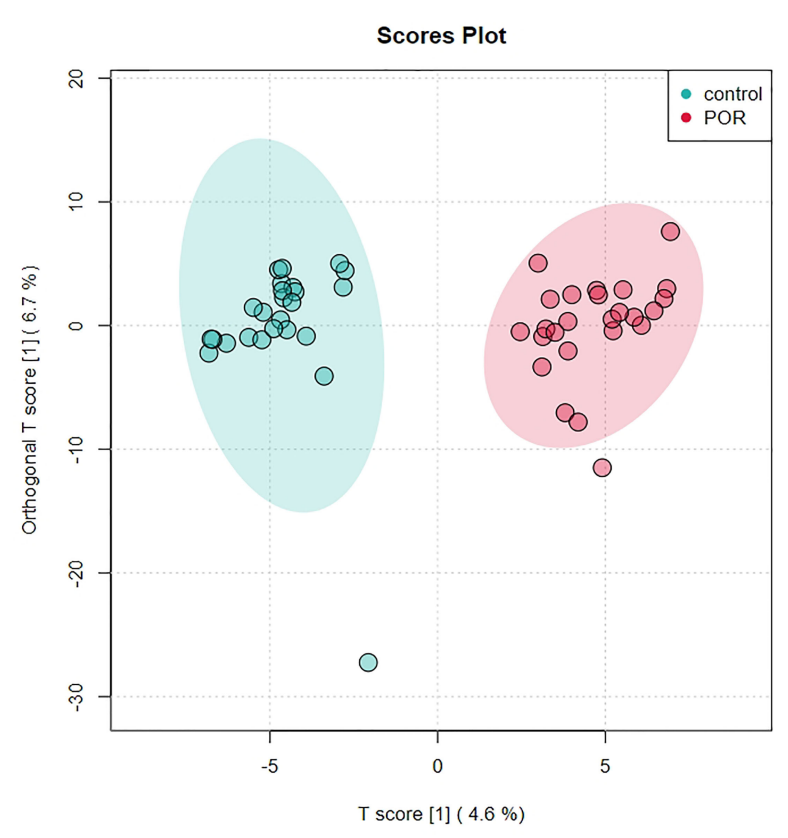

FIGURE 5 | Score plot analysis of OPLS-DA of group of POR and controls. Each point corresponds to a sample. Different groups are marked with different colors, and the region marked by ellipse is the $95 \%$ confidence region of sample points.

\section{Univariate Analysis of Metabolite Differences}

Assessing the impact of changes in metabolites on organisms is necessary, not only knowing whether the changes exist. We can measure the changes by calculating the fold change (FC) of metabolites, and select some important metabolites by combining $p$ values. The increase multiple is positive and the decrease multiple is negative. As shown in Figure 8A, the metabolites in the yellow area of volcano features were those with $p<0.05$ and FC $>2$. These metabolites had significant differences between groups, which should be paid attention to.

In order to intuitively show the differences of metabolites between groups, we made a box chart of the top representative metabolites (the top 25 with smaller $p$ value) (Figure 8B). Lidocaine, 1-naphthylamine, D-erythrulose1-phosphate, diphenylamine, 2-arachidonoylglycerol, pyracarbolid, lanosterin, pelargonic acid, sebacic acid, and 2,4-dinitrophenol were upregulated in the POR group compared with the control group $(p<0.001)$. Prostaglandin H2, 5-hydroxymethyl-2furancarboxaldehyde, cortexolone, and tetracosanoic acid were downregulated in the POR group compared with the control group $(p<0.01)$.

\section{Random Forest Analysis and Support Vector Machines}

In random forest analysis, we used "mean decrease accuracy" and "mean decrease gini" to measure the importance of a metabolite. If the value of a metabolite was changed into a random number, the decrease degree of prediction accuracy of random forest was "mean decrease accuracy"; "mean decrease gini" was the effect of a metabolite on the heterogeneity of observations at all nodes of the classification tree. The larger the two values were, the more important the metabolites were in the random forest. Figure 9A shows the 15 most important metabolites in the random forest, which were quite different between groups.

The closer the area under the curve (AUC) was to 1, the higher the accuracy and specificity was, and the better the predicted effect

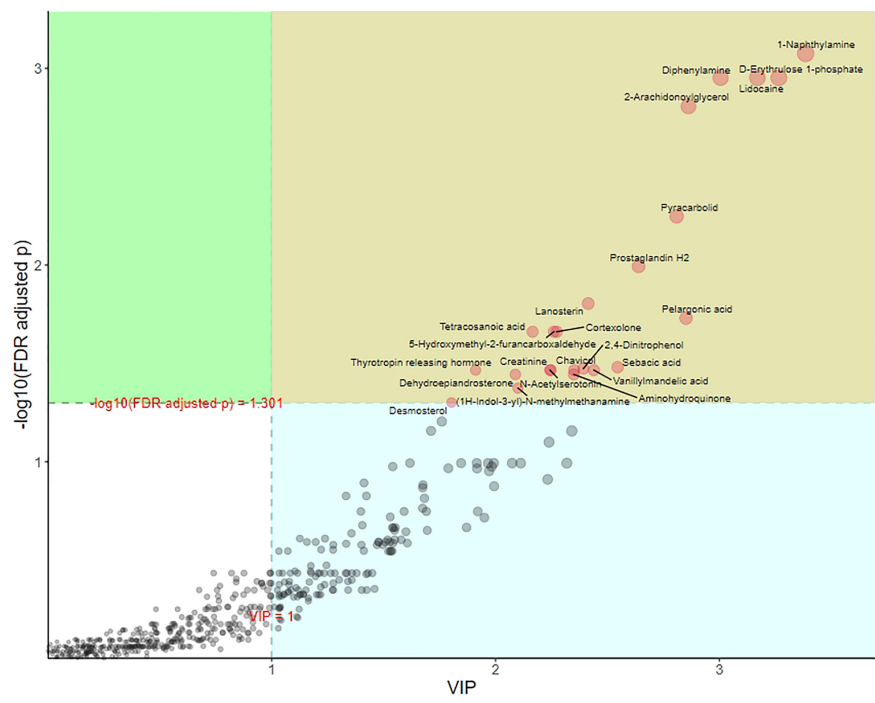

FIGURE 6 | OPLS-DA metabolite importance map of group of POR and controls. Each point represents a metabolite. The abscissa is VIP, and the ordinate is the $p$ value after FDR correction (log10 transformation). 


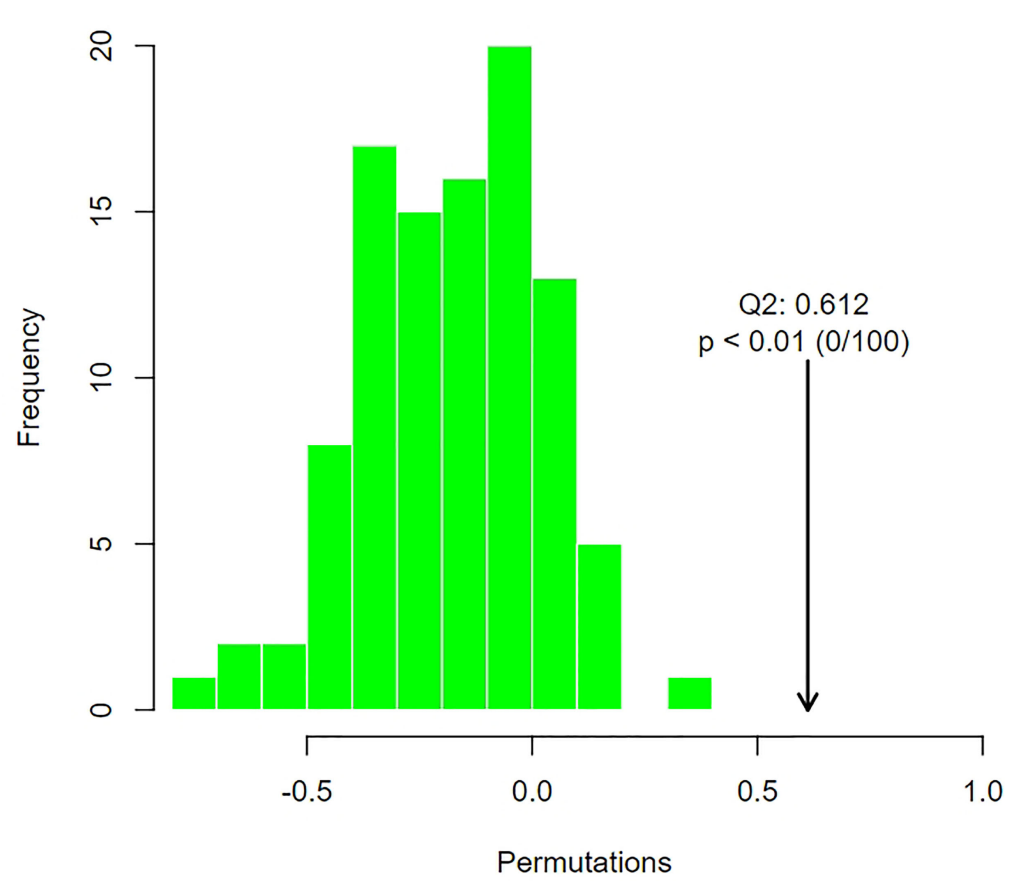

FIGURE 7 | Distribution of test statistics $\left(\mathrm{Q}^{2}\right)$ and $\mathrm{P}$ value of OPLS-DA permutation test of group of POR and controls. The distribution diagram is the permutation random distribution of $Q^{2}$, and the arrow refers to the actually observed model $Q^{2}$.

of the model (21). Figure 9B shows that the better predictions of the model and the greater differences of metabolites were between groups.

Combining the bagging method with SVM, we listed the 15 most important metabolites in SVM, which were significantly different between groups (Figure 9C). They were the thyrotropin releasing hormone, desmosterol, tetracosanoic acid, lidocaine, 2arachidonoylglycerol, 9Z-hexadecenoic acid, cortexolone, prostaglandin H2, 1-naphthylamine, 5-aminopentanoic acid, 5hydroxymethyl-2-furancarboxaldehyde, D-erythrulose1phosphate, $1 \mathrm{H}$-indol-3-yl-N-methylmethanamine, lanosterin, and 2,4-dinitrophenol.

\section{Topological Pathway Enrichment of Metabolites}

The purpose of enrichment analysis is to find the biological pathway which plays a key role in a biological process, so as to reveal and understand the basic molecular mechanism of the biological process (22). Figure 10A shows the metabolic pathway of significant enrichment of different metabolites $(p<0.05)$, which might be of great significance in POR. We listed six metabolic pathways with significant enrichment, which were nicotinate and nicotinamide metabolism, steroid hormone biosynthesis, tyrosine metabolism, vitamin B6 metabolism, butanoate metabolism, and phenylalanine metabolism.

Topological analysis calculated the effect of significant metabolites in metabolic pathways (measured by the impact). Figure 10B shows that the metabolic pathways in the blue region were significant in the over-representation analysis (ORA) enrichment analysis, and the ordinate showed the impact of nicotinate and nicotinamide metabolism in the topological analysis.

Figure 10C shows the metabolic pathway gene map. The color corresponds to the group, indicating that the content of metabolites in the corresponding group is significantly higher (compared with the other group). In the nicotinate and nicotinamide metabolism pathway, L-aspartic acid and 6hydroxynicotinate in the POR group were significantly higher than in the control group, while maleic acid and succinic acid semialdehyde in the POR group were significantly lower than in the control group.

\section{DISCUSSION}

Metabolites refer to the substances produced or consumed through metabolic processes, excluding biological macromolecules. Theoretically, metabolites should include nucleic acids, proteins, lipids, and other small molecular metabolites. The metabolome refers to the dynamic whole of endogenous metabolites. Metabolomics is a new branch of science that qualitatively and quantitatively analyzes all low molecular weight metabolites of an organism or cell in a specific physiological period (20). Metabolomics research can be divided into untargeted and targeted. Untargeted metabolomics can detect all detectable metabolite molecules in the sample without bias, and conduct difference analysis and 


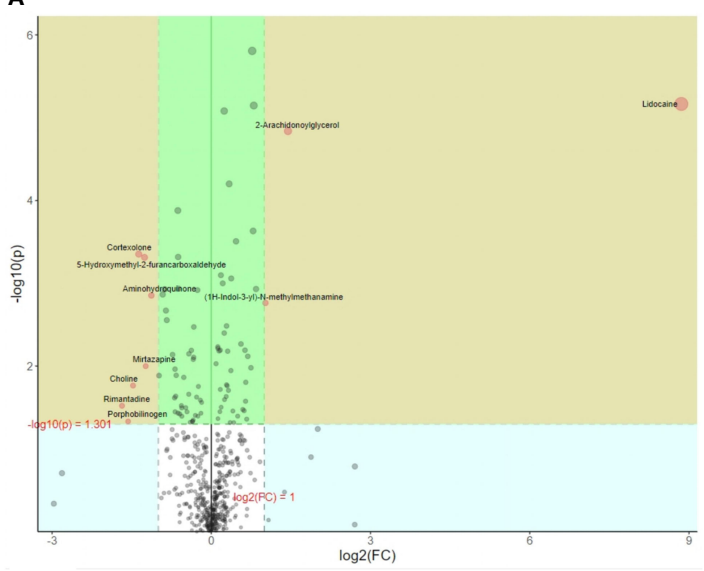

B

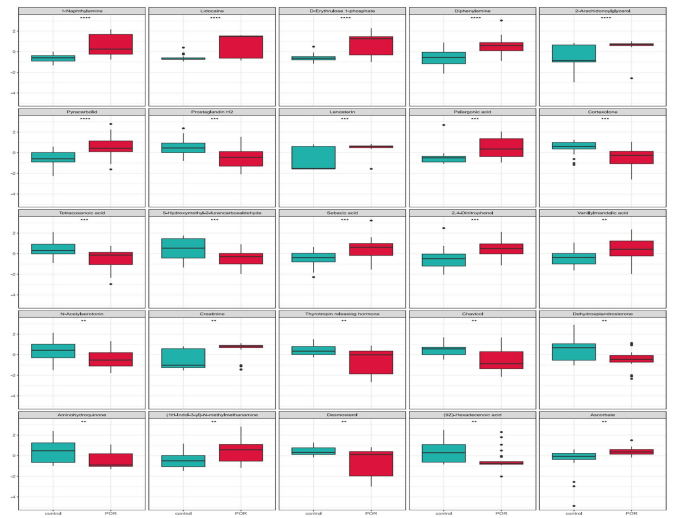

FIGURE 8 | Volcano features (A) and box diagram (B) of metabolite differences. ${ }^{\star \star} p<0.01,{ }^{\star \star \star} p<0.001,{ }^{\star \star \star \star} p<0.0001$.

pathway analysis through the bioinformatics method to find metabolomic biomarkers (23).

POR is a cause of infertility, and it infers a low natural pregnancy rate and poor pregnancy outcome of in vitro fertilization (IVF). Predictive markers and appropriate pretreatment and treatment plans of POR may help clinicians improve the pregnancy rate in a short time and at a low cost (24). Researchers have made many efforts in this area, such as the formulation of the Poseidon classification (25), which comprehensively considered the age, the ovarian reserve markers (AMH and AFC), and responsiveness of POR patients.

In this study, we investigated the relationship between metabolites and physiological and pathological changes by using untargeted metabolomics to study the differences of metabolite expression, providing some potential biomarkers and metabolic pathways for the prediction of POR.

In our study, the clinical characteristics of 25 POR women and 25 control women with matched age had statistically significant differences in the levels of FSH, AMH, and AFC ( $p<0.001)$, which was consistent with other studies $(26,27)$. The differences in age, $\mathrm{LH}, \mathrm{E}_{2}$, and $\mathrm{T}$ in both groups were not significant $(p>0.05)$. PRL in the POR group was significantly

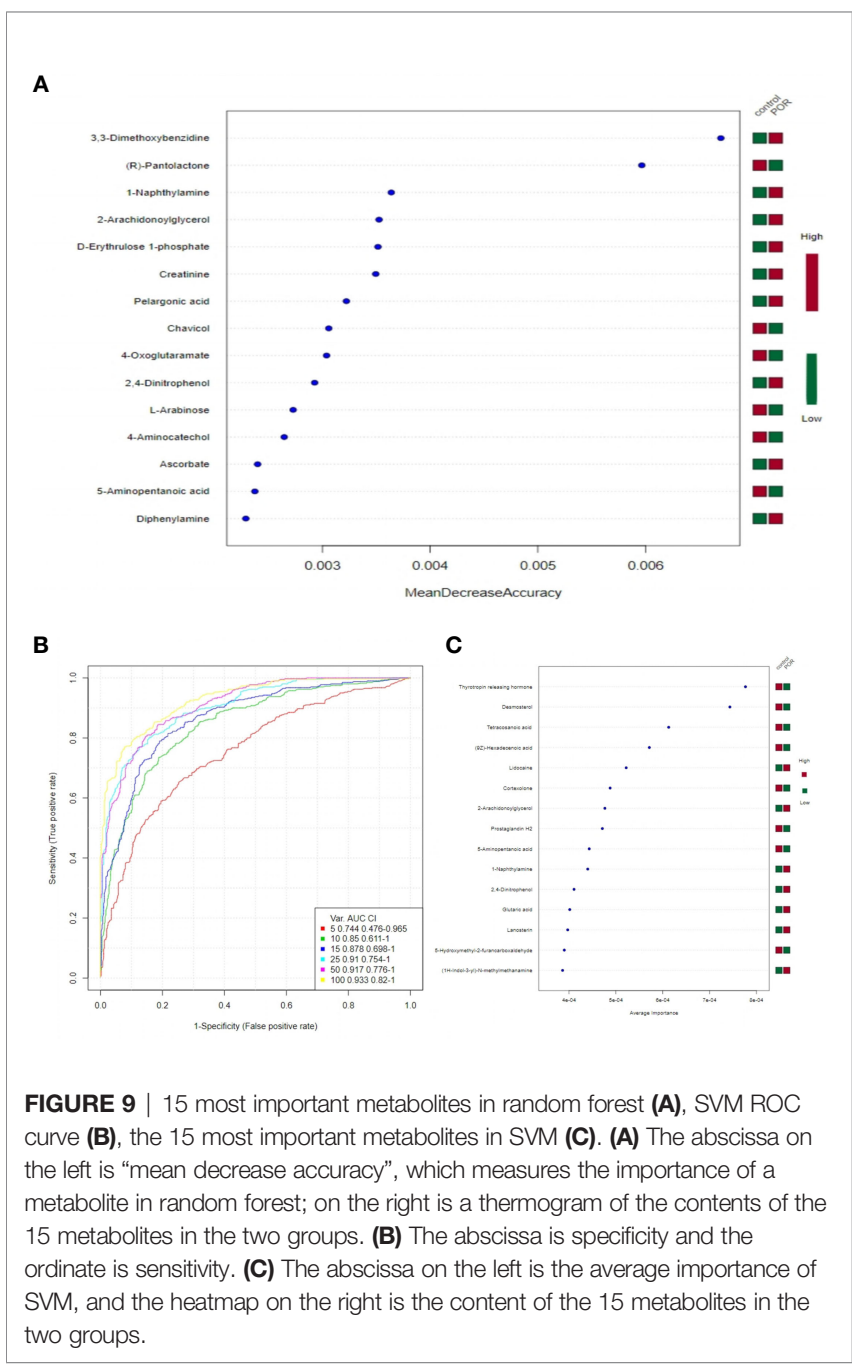

lower than in the control group $(p<0.05)$, which was little analyzed in another POR study (28). There are many influencing factors in $\mathrm{PRL}$, which may have no clinical significance in the prediction markers of POR.

In our study, after the QC and standardization of plasma sample data, we identified 538 metabolites with significant differences in the two groups. Our results showed that POR had significantly higher expressions of 1-naphthylamine, lidocaine, D-erythrulose1phosphate, diphenylamine, 2-arachidonoylglycerol, pyracarbolid, lanosterin, pelargonic acid, sebacic acid, and 2,4-dinitrophenol than the control group $(p<0.001)$. POR had significantly lower expressions of prostaglandin $\mathrm{H} 2$, cortexolone, tetracosanoic acid and 5-hydroxymethyl-2-furancarboxaldehyde than the control group $(p<0.01)$. Combining univariate analysis and the bagging method with SVM, we listed the nine most important metabolites with significant differences (Figure 9C), which were tetracosanoic acid, 2-arachidonoylglycerol, lidocaine, cortexolone, prostaglandin H2, 1-naphthylamine, 5-hydroxymethyl-2-furancarboxaldehyde, D-erythrulose1-phosphate, and 2,4-dinitrophenol $(p<0.05)$. Combining metabolomic profiling together with SVM and pathway analysis, the nicotinate and nicotinamide metabolism pathway, including L-aspartic acid, 6-hydroxynicotinate, maleic 


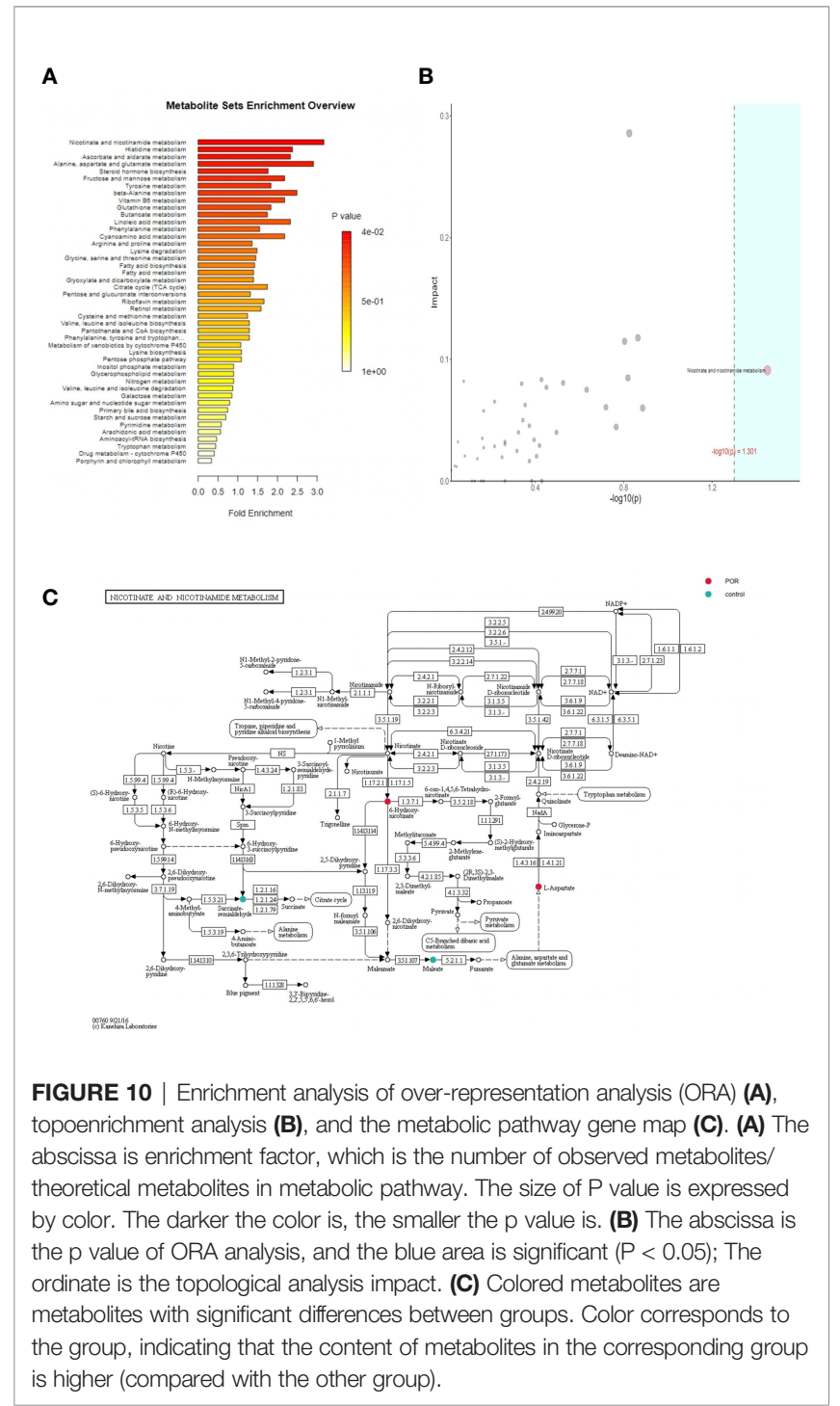

acid, and succinic acid semialdehyde, was identified to have a significant difference in POR women compared to control women $(p<0.05)$, which may be associated with ovarian reserve.

It can be seen from the metabolic network diagram that the nicotinate and nicotinamide metabolism pathway is mainly related to ovarian reserve.

Nicotinate and nicotinamide metabolism has been widely studied in many fields, such as disease treatments and stem cell applications. Nicotinic acid and nicotinamide are two forms of water-soluble B vitamin B3, also known as vitamin PP. As the basic components of coenzymes, they participate in body synthesis and catabolism, and have important effects on animal carbohydrate, lipid, and protein metabolism, and regulate oxidative stress (29). Nicotinamide riboside (NR) treatment in aging mice can delay the degeneration of muscle, enhance muscle function, and increase mouse life span (30). Nicotinamide, as an inhibitor of multiple kinases, can affect the pluripotency and differentiation of human embryonic stem cells (31). Nicotinic acid has long been believed to have a favorable effect on plasma lipids, lowering plasma low density lipoprotein (LDL) and raising high density lipoprotein (HDL) (32). NR supplementation can enhance oxidative metabolism by activating SIRT1 and SIRT3 in high-fat diet-induced mammalian cells and mouse tissues, and improve metabolic abnormalities (33). Excess niacin consumption may cause increased appetite, involve oxidative stress, and increase the prevalence of obesity in US children (34).

Nicotinate and nicotinamide metabolism has also been studied in obstetrics and gynecology. One animal study showed different concentrations of niacin in the medium could impact the maturation quality of in vitro embryo production (IVP) embryos and total tolerance of bovine oocytes to vitrification (35). Polycystic ovary syndrome (PCOS) is one of the common reproductive endocrine diseases for fertility in women of reproductive age. The clinical manifestations of PCOS are highly heterogeneous, including sparse ovulation or prolonged anovulation, hyperandrogenemia, insulin resistance, infertility, dyslipidemia, and obesity. For PCOS, additional supplementation of B1, folates with inositol, niacinamide, and antioxidants in the diet should be recommended to improve insulin sensitivity (36). Nicotinamide might be partially mediated in an adenosine 5'-monophosphate (AMP)-activated protein kinase (AMPK) manner, and was found to reduce the serum testosterone levels and CYP17A1 gene expression in a letrozole-induced rat model of PCOS (37).

Administration of niacin promoted follicle growth by increasing germ-line cell marker DDX4 and cell proliferation marker PCNA in the ovary, inhibited apoptosis in vitro, and treated premature ovarian failure (POF) in mouse models, which were under harmful conditions, such as radiation and chemotherapy damage (38). It was reported that consistent administration of nicotinamide with appropriate concentrations could maintain DNA integrity and prevent the progression of programmed cell death (PCD) (39). Nicotinamide as a dietary supplement might protect cells against apoptosis induced by sodium deoxycholate ( $\mathrm{NaDOC}$ ), independent of oxidative stress (40). Imidacloprid (IMI) is a worldwide highest selling insecticide, which is more toxic to the mammalian nervous system. 6-hydroxynicotinic acid, as one of the major metabolites of IMI, was observed in female rats where the concentration was higher in the ovary and blood after oral administration of IMI (41). In this study, 6-hydroxynicotinic acid was higher in POR than controls, which may show that 6-hydroxynicotinic acid is toxic to the ovary. POR women may contact more IMI or other hazardous substances and environmental exposure than controls. N1-methylnicotinamide was reported to postpone aging based on the activation of the ovarian AMPK in a rat model of PCOS (42). Some metabolisms in the nicotinate and nicotinamide metabolism pathway may play an important role in POR.

\section{CONCLUSION}

In our present study, the metabolic alterations in POR and normal patients were characterized using an LC-MS-based 
metabolomics approach. Clear metabolic differences were observed between POR and controls. These variations involved significant perturbations in glycerophospholipid, glycerolipid, and fatty acid metabolisms. The nicotinate and nicotinamide metabolism pathway may offer new insight for complementary prediction and treatment of POR. It is important to pre-assess the ovary function of women of childbearing age and adopt an individualized scheme for their family planning. However, this study had some limitations as well. The sample size included was small, and the research results may be biased to some extent. The functional associations of this metabolomics in POR need further investigation. Our findings also suggest that LC-MS-based metabolomics research offers a promising strategy for the complementary diagnosis of POR. The counterbalance of the corresponding molecular events might contribute to the prediction, alleviation, and treatment of POR.

\section{STRENGTHS AND LIMITATIONS}

Our results demonstrated that the nicotinate and nicotinamide metabolism pathway had an important effect in POR using the untargeted LC-MS metabonomic method. There were some limitations in this research including the small sample size and the lack of an underlying mechanism. In the future, first, we should plan to conduct more research with a sufficient number of participants. Next, we should conduct cell experiments and animal experiments to verify the effect and pathogenesis of this pathway in POR.

\section{REFERENCES}

1. Ferraretti AP, La Marca A, Fauser BC, Tarlatzis B, Nargund G, Gianaroli L. ESHRE Working Group on Poor Ovarian Response Definition. ESHRE Consensus on the Definition of 'Poor Response' to Ovarian Stimulation for In Vitro Fertilization: The Bologna Criteria. Hum Reprod (2011) 26(7):161624. doi: 10.1093/humrep/der092

2. Poseidon Group (Patient-Oriented Strategies Encompassing IndividualizeD Oocyte Number), Alviggi C, Andersen CY, Buehler K, Conforti A, De Placido G, et al. A New More Detailed Stratification of Low Responders to Ovarian Stimulation: From a Poor Ovarian Response to a Low Prognosis Concept. Fertil Steril (2016) 105(6):1452-3. doi: 10.1016/j.fertnstert.2016.02.005

3. Hansen KR, Craig LB, Zavy MT, Klein NA, Soules MR. Ovarian Primordial and Nongrowing Follicle Counts According to the Stages of Reproductive Aging Workshop (STRAW) Staging System. Menopause (2012) 19(2):164-71. doi: 10.1097/gme.0b013e31823b0b2e

4. Iliodromiti S, Iglesias Sanchez C, Messow CM, Cruz M, Garcia Velasco J, Nelson SM. Excessive Age-Related Decline in Functional Ovarian Reserve in Infertile Women: Prospective Cohort of 15,500 Women. J Clin Endocrinol Metab (2016) 101(9):3548-54. doi: 10.1210/jc.2015-4279

5. Jayaprakasan K, Campbell B, Hopkisson J, Johnson I, Raine-Fenning N. A Prospective, Comparative Analysis of Anti-Müllerian Hormone, Inhibin-B, and Three-Dimensional Ultrasound Determinants of Ovarian Reserve in the Prediction of Poor Response to Controlled Ovarian Stimulation. Fertil Steril (2010) 93(3):855-64. doi: 10.1016/j.fertnstert.2008.10.042

6. Xu H, Feng G, Wang H, Han Y, Yang R, Song Y, et al. A Novel Mathematical Model of True Ovarian Reserve Assessment Based on Predicted Probability of Poor Ovarian Response: A Retrospective Cohort Study. J Assist Reprod Genet (2020) 37(4):963-72. doi: 10.1007/s10815-020-01700-1

\section{DATA AVAILABILITY STATEMENT}

The original contributions presented in the study are included in the article/supplementary materials. Further inquiries can be directed to the corresponding authors.

\section{ETHICS STATEMENT}

The studies involving human participants were reviewed and approved by the Ethics Committee of Shanxi Provincial People's Hospital. The patients/participants provided their written informed consent to participate in this study. Written informed consent was obtained from the individual(s) for the publication of any potentially identifiable images or data included in this article.

\section{AUTHOR CONTRIBUTIONS}

HS designed the main study. CY, LF, and FZ contributed to data collection. HS and HL performed the statistical analysis and interpreted the results. HS wrote the manuscript. QQ contributed to the critical revision of the article. All authors reviewed and approved the final version.

\section{FUNDING}

The National Natural Science Foundation of China (81401192) supported QQ for this study.

7. Kaddurah-Daouk R, Kristal BS, Weinshilboum RM. Metabolomics: A Globa Biochemical Approach to Drug Response and Disease. Annu Rev Pharmacol Toxicol (2008) 48:653-83. doi: 10.1146/annurev.pharmtox.48.113006.094715

8. Dunn WB, Broadhurst D, Begley P, Zelena E, Francis-McIntyre S, Anderson N, et al. Procedures for Large-Scale Metabolic Profiling of Serum and Plasma Using Gas Chromatography and Liquid Chromatography Coupled to Mass Spectrometry. Nat Protoc (2011) 6(7):1060-83. doi: 10.1038/nprot.2011.335

9. Zelena E, Dunn WB, Broadhurst D, Francis-McIntyre S, Carroll KM, Begley P, et al. Development of a Robust and Repeatable UPLC-MS Method for the Long-Term Metabolomic Study of Human Serum. Anal Chem (2009) 81 (4):1357-64. doi: 10.1021/ac8019366

10. Sangster T, Major H, Plumb R, Wilson AJ, Wilson ID. A Pragmatic and Readily Implemented Quality Control Strategy for HPLC-MS and GC-MSBased Metabonomic Analysis. Analyst (2006) 131(10):1075-8. doi: 10.1039/ b604498k

11. Buzatto AZ, de Sousa AC, Guedes SF, Cieslarová Z, Simionato AV. Metabolomic Investigation of Human Diseases Biomarkers by CE and LC Coupled to MS. Electrophoresis (2014) 35(9):1285-307. doi: 10.1002/ elps.201300470

12. Zhou Y, Song R, Ma C, Zhou L, Liu X, Yin P, et al. Discovery and Validation of Potential Urinary Biomarkers for Bladder Cancer Diagnosis Using a Pseudotargeted GC-MS Metabolomics Method. Oncotarget (2017) 8 (13):20719-28. doi: 10.18632/oncotarget.14988

13. Falegan OS, Ball MW, Shaykhutdinov RA, Pieroraio PM, Farshidfar F, Vogel HJ, et al. Urine and Serum Metabolomics Analyses May Distinguish Between Stages of Renal Cell Carcinoma. Metabolites (2017) 7(1):6. doi: 10.3390/ metabo7010006

14. Claudino WM, Quattrone A, Biganzoli L, Pestrin M, Bertini I, Di Leo A. Metabolomics: Available Results, Current Research Projects in Breast Cancer, 
and Future Applications. J Clin Oncol (2007) 25(19):2840-6. doi: 10.1200/ JCO.2006.09.7550

15. Atiomo W, Daykin CA. Metabolomic Biomarkers in Women With Polycystic Ovary Syndrome: A Pilot Study. Mol Hum Reprod (2012) 18(11):546-53. doi: $10.1093 / \mathrm{molehr} / \mathrm{gas} 029$

16. Zhang XJ, Huang LL, Su H, Chen YX, Huang J, He C, et al. Characterizing Plasma Phospholipid Fatty Acid Profiles of Polycystic Ovary Syndrome Patients With and Without Insulin Resistance Using GC-MS and Chemometrics Approach. J Pharm BioMed Anal (2014) 95:85-92. doi: 10.1016/j.jpba.2014.02.014

17. Dong F, Deng D, Chen H, Cheng W, Li Q, Luo R, et al. Serum Metabolomics Study of Polycystic Ovary Syndrome Based on UPLC-QTOF-MS Coupled With a Pattern Recognition Approach. Anal Bioanal Chem (2015) 407 (16):4683-95. doi: 10.1007/s00216-015-8670-x

18. Chong J, Xia J. MetaboAnalystR: An R Package for Flexible and Reproducible Analysis of Metabolomicsdata. Bioinformatics (2018) 34(24):4313-4. doi: 10.1093/bioinformatics/bty528

19. Westerhuis JA, van Velzen EJ, Hoefsloot HC, Smilde AK. Multivariate Paired Data Analysis: Multilevel PLSDA Versus OPLSDA. Metabolomics (2010) 6 (1):119-28. doi: 10.1007/s11306-009-0185-z

20. Nordström A, O'Maille G, Qin C, Siuzdak G. Nonlinear Data Alignment for UPLC-MS and HPLC-MS Based Metabolomics: Quantitative Analysis of Endogenous and Exogenous Metabolites in Human Serum. Anal Chem (2006) 78(10):3289-95. doi: 10.1021/ac060245f

21. Moore RG, Brown AK, Miller MC, Skates S, Allard WJ, Verch T, et al. The Use of Multiple Novel Tumor Biomarkers for the Detection of Ovarian Carcinoma in Patients With a Pelvic Mass. Gynecol Oncol (2008) 108(2):402-8. doi: 10.1016/j.ygyno.2007.10.017

22. Christensen CD, Hofmeyr JS, Rohwer JM. PySCeSToolbox: A Collection of Metabolic Pathway Analysis Tools. Bioinformatics (2018) 34(1):124-5. doi: 10.1093/bioinformatics/btx567

23. Peng J, Chen YT, Chen CL, Li L. Development of a Universal Metabolomestandard Method for Long-Term LC-MS Metabolome Profiling and Its Application for Bladder Cancer Urine-Metabolite-Biomarker Discovery. Anal Chem (2014) 86:6540-7. doi: 10.1021/ac5011684

24. La Marca A, Sunkara SK. Individualization of Controlled Ovarian Stimulation in IVF Using Ovarian Reserve Markers: From Theory to Practice. Hum Reprod Update (2014) 20(1):124-40. doi: 10.1093/humupd/dmt037

25. Grisendi V, Mastellari E, La Marca A. Ovarian Reserve Markers to Identify Poor Responders in the Context of Poseidon Classification. Front Endocrinol (Lausanne) (2019) 10:281. doi: 10.3389/fendo.2019.00281

26. Fleming R, Seifer DB, Frattarelli JL, Ruman J. Assessing Ovarian Response: Antral Follicle Count Versus Anti-Müllerian Hormone. Reprod BioMed Online (2015) 31(4):486-96. doi: 10.1016/j.rbmo.2015.06.015

27. La Marca A, Sighinolfi G, Radi D, Argento C, Baraldi E, Artenisio AC, et al. Anti-Mullerian Hormone (AMH) as a Predictive Marker in Assisted Reproductive Technology (ART). Hum Reprod Update (2010) 16(2):113-30. doi: 10.1093/humupd/dmp036

28. Tal R, Seifer DB. Ovarian Reserve Testing: A User's Guide. Am J Obstet Gynecol (2017) 217(2):129-40. doi: 10.1016/j.ajog.2017.02.027

29. Bogan KL, Brenner C. Nicotinic Acid, Nicotinamide, and Nicotinamide Riboside: A Molecular Evaluation of NAD+ Precursor Vitamins in Human Nutrition. Annu Rev Nutr (2008) 28:115-30. doi: 10.1146/annurev.nutr. 28.061807.155443

30. Zhang H, Ryu D, Wu Y, Gariani K, Wang X, Luan P, et al. NAD ${ }^{+}$Repletion Improves Mitochondrial and Stem Cell Function and Enhances Life Span in Mice. Science (2016) 352(6292):1436-43. doi: 10.1126/science.aaf2693

31. Meng Y, Ren Z, Xu F, Zhou X, Song C, Wang VY, et al. Nicotinamide Promotes Cell Survival and Differentiation as Kinase Inhibitor in Human
Pluripotent Stem Cells. Stem Cell Rep (2018) 11(6):1347-56. doi: 10.1016/ j.stemcr.2018.10.023

32. Altschul R, Hoffer A, Stephen JD. Influence of Nicotinic Acid on Serum Cholesterol in Man. Arch Biochem Biophys (1955) 54(2):558-9. doi: 10.1016/ 0003-9861(55)90070-9

33. Cantó C, Houtkooper RH, Pirinen E, Youn DY, Oosterveer MH, Cen Y, et al. The NAD $(+)$ Precursor Nicotinamide Riboside Enhances Oxidative Metabolism and Protects Against High-Fat Diet-Induced Obesity. Cell Metab (2012) 15(6):838-47. doi: 10.1016/j.cmet.2012.04.022

34. Li D, Sun WP, Zhou YM, Liu QG, Zhou SS, Luo N, et al. Chronic Niacin Overload may be Involved in the Increased Prevalence of Obesity in US Children. World $J$ Gastroenterol (2010) 16(19):2378-87. doi: 10.3748/wjg.v16.i19.2378

35. Kafi M, Ashrafi M, Azari M, Jandarroodi B, Abouhamzeh B, Asl AR. Niacin Improves Maturation and Cryo-Tolerance of Bovine In Vitro Matured Oocytes: An Experimental Study. Int J Reprod Biomed (2019) 17(9):621-8. doi: $10.18502 /$ ijrm.v17i9.5096

36. Szczuko M, Szydłowska I, Nawrocka-Rutkowska J. A Properly Balanced Reduction Diet and/or Supplementation Solve the Problem With the Deficiency of These Vitamins Soluble in Water in Patients With PCOS. Nutrients (2021) 13(3):746. doi: 10.3390/nu13030746

37. Nejabati HR, Samadi N, Shahnazi V, Mihanfar A, Fattahi A, Latifi Z, et al. Nicotinamide and its Metabolite N1-Methylnicotinamide Alleviate Endocrine and Metabolic Abnormalities in Adipose and Ovarian Tissues in Rat Model of Polycystic Ovary Syndrome. Chem Biol Interact (2020) 324:109093. doi: 10.1016/j.cbi.2020.109093

38. Wang S, Sun M, Yu L, Wang Y, Yao Y, Wang D. Niacin Inhibits Apoptosis and Rescues Premature Ovarian Failure. Cell Physiol Biochem (2018) 50 (6):2060-70. doi: 10.1159/000495051

39. Lin SH, Vincent A, Shaw T, Maynard KI, Maiese K. Prevention of Nitric Oxide-Induced Neuronal Injury Through the Modulation of Independent Pathways of Programmed Cell Death. J Cereb Blood Flow Metab (2000) 20 (9):1380-91. doi: 10.1097/00004647-200009000-00013

40. Crowley CL, Payne CM, Bernstein H, Bernstein C, Roe D. The NAD+ Precursors, Nicotinic Acid and Nicotinamide Protect Cells Against Apoptosis Induced by a Multiple Stress Inducer, Deoxycholate. Cell Death Differ (2000) 7(3):314-26. doi: 10.1038/sj.cdd.4400658

41. Kapoor U, Srivastava MK, Trivedi P, Garg V, Srivastava LP. Disposition and Acute Toxicity of Imidacloprid in Female Rats After Single Exposure. Food Chem Toxicol (2014) 68:190-5. doi: 10.1016/j.fct.2014.03.019

42. Nejabati HR, Schmeisser K, Shahnazi V, Samimifar D, Faridvand Y, BahramiAsl Z, et al. N1-Methylnicotinamide: An Anti-Ovarian Aging Hormetin? Ageing Res Rev (2020) 62:101131. doi: 10.1016/j.arr.2020.101131

Conflict of Interest: The authors declare that the research was conducted in the absence of any commercial or financial relationships that could be construed as a potential conflict of interest.

Publisher's Note: All claims expressed in this article are solely those of the authors and do not necessarily represent those of their affiliated organizations, or those of the publisher, the editors and the reviewers. Any product that may be evaluated in this article, or claim that may be made by its manufacturer, is not guaranteed or endorsed by the publisher.

Copyright (c) 2021 Song, Qin, Yuan, Li, Zhang and Fan. This is an open-access article distributed under the terms of the Creative Commons Attribution License (CC BY). The use, distribution or reproduction in other forums is permitted, provided the original author(s) and the copyright owner(s) are credited and that the original publication in this journal is cited, in accordance with accepted academic practice. No use, distribution or reproduction is permitted which does not comply with these terms. 